\title{
Pricing Onion Options: A Probabilistic Approach
}

\author{
Thorsten Upmann ${ }^{1}$ \\ ${ }^{1}$ Mercator School of Management, University Duisburg-Essen, Duisburg, Germany \\ Correspondence: Thorsten Upmann, Mercator School of Management, University Duisburg-Essen, Lotharstraße 65, \\ 47057 Duisburg, Germany. Tel: 49-203-379-1440. E-mail: Thorsten.Upmann@uniduisburg-essen.de
}

Received: June 5, 2013

Accepted: June 27, 2013

Online Published: October 8, 2013

doi:10.5430/ijfr.v4n4p11

URL: http://dx.doi.org/10.5430/ijfr.v4n4p11

The publication of this article is financially supported by the programme "Open Access Publizieren" of the Deutsche Forschungsgemeinschaft (German Research Foundation). This support is gratefully acknowledged.

\begin{abstract}
As argued by Ebenfeld, Mayr and Topper (2002), Onion options may be decomposed into one-touch double barrier binary options (ODBs). Using this idea, these authors provide an arbitrage-free pricing formula for Onion options within the Black-Scholes framework. Their approach rests upon solving the underlying partial differential equation. In this paper, we take an alternative and more direct route: Based on a probabilistic approach, we compute the risk-neutral valuation formula for an ODB. Then, by inverting the decomposition of an Onion option, we are able to derive an alternative pricing formula for this type of an option.
\end{abstract}

Keywords: onion options, one-touch double barrier binary options, double-notouch options, arbitrage-free pricing, Black-Scholes model

\section{Introduction}

Among the vast group of Exotic Options the so called Onion options gained some popularity, (Note 1) and as a consequence, they became regularly traded at, for example, the Deutsche B" orse in Frankfurt, Germany and at the stock exchange in Stuttgart, Germany. Onion options may be viewed as nested digital double-barrier options. That is, they are composed of a couple of digital knock-out options each of which is characterized by a corridor for the price of the underlying. Ebenfeld, Mayr and Topper (2002) used this composition of Onion options in order to derive an arbitrage-free pricing formula. More precisely, they proposed to decompose such an option into a series of so-called one-touch double barrier binary options (ODBs). Then, after deriving an arbitrage-free price for an ODB, the linearity of the pricing rule (i. e.. the fundamental theorem of finance) may then be applied to obtain an arbitrage-free price for an Onion option. In order to find a valuation formula for an ODB, Ebenfeld, Mayr and Topper (2002) applied the classical Black-Scholes model. In particular, they used the Black-Scholes partial differential equation (PDE) in order to derive an arbitrage-free pricing formula for an ODB - and thus for an Onion option. In fact, it can be shown that this formula is the unique solution of the PDE and thus the unique value for an Onion option in the Black-Scholes model.

In this paper we provide an alternative approach to the derivation of a valuation formula for an Onion option. While Ebenfeld, Mayr and Topper, after suitable transformation of variables, transformed the Black-Scholes PDE into the well known heat equation, we follow a completely different approach here, which we believe to be more direct and thus more intuitive: It is based on a probabilistic approach by computing the risk-neutral valuation formula for currency options. A similar procedure is also pursued by Geman and Yor (1996) who price double barrier options via a probabilistic approach. Similarly, Kunitomo and Ikeda (1992) price double barrier options with curved boundaries via a suitable probability measure.

More recently, the pricing of double barrier options has advanced, and correspondingly, the amount of academic research on this class of options has surged substantially. For example, Luo (2001) derives closed-form solutions for eight types of European-style double-barrier options. Guillaume (2003) examines window double barrier option (options where the monitoring period starts after the beginning of the contract and terminates before its expiry). Labart and Lelong (2009) study double barrier Parisian options, options where the payoff condition depends on the time spent in a row above or below a barrier (or a series of barriers), and not just on the hitting time(s). Buchen (2009) 
considers pricing of double knock-out barrier options with arbitrary payoff functions and time-varying barriers. Chen, Wang and Shyu (2010) apply the reflection principle to derive a close-form pricing formula of double barrier-options. Guillaume (2010) derives a pricing formula for double barrier options where the barrier is a step function of time hence called step double barrier options. And most recently, an analysis of hedging strategies of (digital) double barrier options is provided by Obloj and Ulmer (2012).

\section{Onion Options and Related Derivatives}

Onion options may be viewed as a particular type of nested barrier options. (Note 2) To begin with, consider a double barrier option, which is a simple example for a non-standard barrier option. This type of option combines an upper with a lower barrier which are effective simultaneously. Therefore, to trigger either the knockout or the knock-in effect, it is sufficient that the price of the underlying reaches either of both barriers.

The Onion option is a generalized double barrier option: It consists of a couple of pairs of barriers, each of which is composed of a lower barrier $\mathrm{H}^{-}$and an upper barrier $\mathrm{H}^{+}$which lies below respectively above the initial spot price of the underlying. In order to ease exposition, we subsequently assume that there are exactly three couples of barriers, denoted by $H_{i}^{-}$and $H_{i}^{+}, i=1,2,3$. Every couple $\left(H_{i}^{-}, H_{i}^{+}\right)$builds a corridor around the initial price of the underlying, such that

$$
H_{3<}^{-}<H_{2}^{-}<H_{1<}^{-} S_{0}<H_{1<}^{+} H_{2}^{+}<H_{3}^{+}
$$

holds, where $S_{0}$ denotes the initial spot price of the underlying. Hence, there is an inner range, a middle range, and an outside range. Basically, these corridors work like barriers of a vanilla knock-out option or, to be more precise, like the corridor of a double barrier option. Therefore, the two barriers of a corridor work simultaneously, meaning that the option, or in this case the corridor, gets knockedout if and when either of the two barriers of the respective corridor is hit by the price of the underlying. - It makes no difference whether the upper or the lower barrier is reached; and once a corridor is knocked-out it can not be knocked in again later.

The (possible) payoff of the option, received at expiry, is a predetermined fixed amount that makes the Onion option similar to a corridor-dependent binary option. More precisely, an Onion option represents a mixture of barrier and cash-or- nothing options (CON). (Note 3) The exact amount depends on the range which the price of the underlying has left during the option's life. Assume, for instance, that each corridor is worth 10 Euro. Then, the maximum payoff is 30 Euro when no range is left. If the inner range is the only one which was left by the price of the underlying during the option's life, the payoff equals 20 Euro. If only the barriers of the third corridor are not reached, a payoff of 10 Euro remains. When this last range is also left, the option vanishes instantly. The payoff of the option thus depends on the minimum and the maximum of the underlying during the lifetime of the option, that is on the running minimum and maximum at maturity, $m(T)$ and $M(T)$ respectively.

Frequently, Onion options are written on currencies, that is on foreign ex-change rates. However, there are a priori no reasons to restrict the type of the underlying to an exchange rate. Nevertheless, in the rest of this text we focus on the currency Onion option.

\section{Decomposing Onion Options}

In the following our goal is to find a pricing formula for the Onion option. In this respect the main problem is how to include all of the barriers into a single formula. The major idea is to split an Onion option with, say, three corridors into three other options which synthetically replicate the onion option. Then, using therisk-neutral valuation formula and finding a suitable probability measure, we are able to derive a valuation formula for the Onion option within the Black-Scholes framework.

To be more precise, we decompose the Onion option into three one-touch double barrier binary options (ODB), also known as double-no-touch digital options or double-lock-out options, and then look for a pricing formula for an ODB. (Note 4) ODBs may be viewed as Onion options with only one corridor or as double barrier options with a binary payoff. Accordingly the two barriers again work simultaneously and, therefore, in order to trigger the knock-out effect it suffices that either of both barriers is reached.

We now decompose the payoff of an Onion option, which allows us to split it into a series of ODB options. From Section 2 we know that the payoff of an Onion option is determined by the barriers of three corridors $H_{i}^{-}, H_{i}^{+}, i=$ $1,2,3$. Let $S_{t}, t \in[0, T]$ stand for the value of the underlying at time $t \in[0, T]$, where $\mathrm{T}$ is the date of maturity. To write the payoff explicitly as a function, we define the following two random variables

$$
m(t):=\min _{0 \leq u \leq t} S(u), \text { and } M(t):=\max _{0 \leq u \leq t} S(u) .
$$


$m(t)$ and $M(t)$ are $\mathcal{F}^{t}$-measurable. The payoff function of the Onion option is denoted by $\psi$ and can be written

as

$$
\psi=\left\{\begin{aligned}
3 R: & H_{1}^{-}<m(T) \leq M(T)<H_{1}^{+} \\
2 R: & H_{2}^{-}<m(T) \leq M(T)<H_{2}^{+} \text {and }\left\{m(T)<H_{1}^{-} \text {or } M(T)>H_{1}^{+}\right\} \\
R: & H_{3}^{-}<m(T) \leq M(T)<H_{3}^{+} \text {and }\left\{m(T)<H_{2}^{-} \text {or } M(T)>H_{2}^{+}\right\} \\
0: & \text { else, }
\end{aligned}\right.
$$

where $R$ denotes the fixed payoff that the holder of the option receives per corridor which the price of the underlying does not leave until maturity. For example, the investor receives $3 R$ Euro when the underlying stays inside the inner corridor during the option's life, that is, the minimum of the underlying in this period must be above the first lower barrier $\mathrm{H}^{-}$and the maximum has to lie below the first upper barrier $\mathrm{H}^{+}$. The two other corridors work analogously.

In order to explain the connection between the Onion option and the ODB options, observe that $\psi$ can be denoted as a sum of the payoff functions of three ODB options

$$
\psi=\psi_{1}+\psi_{2}+\psi_{3},
$$

where the payoff function of an ODB option is given by

$$
\psi_{i}=\left\{\begin{array}{rll}
R & : & H_{i}^{-}<m(T) \leq M(T)<H_{i}^{+} \\
0 & : & \text { else }
\end{array} \quad, \quad \text { for } i=1,2,3 .\right.
$$

Then, formulas (1) and (2) apparently coincide.

A portfolio consisting of three ODB options can thus be considered as a synthetic Onion option if the three corridors are arranged in the same way as the Onion option's three corridors. Specified in this way, the synthetic option must cost the same as the Onion option under the assumption of no arbitrage. Since the Onion option can synthetically be replicated by three ODBs, it is sufficient to find a pricing formula for an ODB option.

\section{Pricing One-Touch Double Barrier Binary Options}

The problem of pricing an Onion option is now reduced to the problem of pricing one-touch double barrier binary options (ODBs). Hui (1996) derives a pricing formula for this kind of an option and this section draws upon his paper. In order to price an ODB, the Black-Scholes framework is utilized (along with the familiar notation). In particular, we apply the Black-Scholes PDE for foreign currency options (see, for example, Musiela and Rutkowski, 2005, ch. 3 and 4; and Bjork, 2009, ch. 17):

$$
\frac{\partial f}{\partial t}+\frac{1}{2} \sigma^{2} S^{2} \frac{\partial^{2} f}{\partial S^{2}}+\left(r_{d}-r_{f}\right) S \frac{\partial f}{\partial S}-r_{d} f=0
$$

which holds for all $(S, t) \in(0, \infty) \times(0, T)$ subject to the terminal condition

$$
f(s, T)=g(s) .
$$

$\left(r_{d}\right.$ and $r_{f}$ denote the domestic and the foreign interest rate, respectively.) First, however, we have to verify whether or not the payoff of an ODB fulfils the requirements of this formula and in particular of the following familiar theorem in order to be applicable to ODB options.

Theorem 4.1 (Black-Scholes PDE) Let $g: \mathbb{R} \rightarrow \mathbb{R}$ be a Borel-measurable function such that the random variable $X=g\left(S_{T}\right)$ is square-integrable under $\mathbb{P}^{*}$. Then the arbitrage price in $\mathcal{M}_{B S}$ of the claim $X$ which settles at time $T$ is given by the equality $\pi_{t}(X)=v\left(S_{t}, t\right)$ where the function $v: \mathbb{R}_{+} \times[0, T] \rightarrow \mathbb{R}_{\text {solves the Black-Scholes partial differential equation }}$ 


$$
\frac{\partial v}{\partial t}+\frac{1}{2} \sigma^{2} s^{2} \frac{\partial^{2} v}{\partial s^{2}}+r s \frac{\partial v}{\partial s}-r v=0,
$$

for all $(s, t) \in(0, \infty) \times(0, T)$, subject to the terminal condition $v(s, T)=g(s)$.

In particular, we have to check whether or not the ODB's payoff function $g(s)$ is measurable and integrable. To this end, we modify the payoff function of an ODB, given by eq. (3), somewhat to obtain:

$$
g(s)=\left\{\begin{array}{rll}
R & : & H^{-}<s<H^{+} \\
0 & : & \text { else }
\end{array},\right.
$$

where $\mathrm{H}^{+}$and $\mathrm{H}^{-}$are the upper and the lower barrier, and $R$ denotes the payoff of the ODB. Obviously, $g$ is a piecewise continuous function with two discontinuities. Therefore, $g$ is measurable and also integrable. Hence, the Black-Scholes PDE is applicable in this case.

Now, we have to argue why it is sufficient to consider the (modified) payoff function $g$ instead of $\psi_{i}$ as given in eq. (3). To see this, recall that the terminal payoff is only paid if the underlying stays inside the corridor during the whole time interval. Thus, today's value of the option is nil if it has already been knocked-out. However, we are only interested in the non-trivial case where the option has not yet been knocked out. So whenever we are looking for the value of an option we are always implicitly assuming that it is still alive. Formally, the dynamic of the pricing formula $f(S, t)$ is only defined on the open set $\left(H^{-}, H^{+}\right) \times(0, T)$. This implies when the open set is left, the dynamic is no longer described by the Black-Scholes PDE but by the boundary conditions. They state that the valuation formula equals zero on the boundary. As the dynamic on the boundary is also given by these conditions, they imply that it will stay on the boundary for the rest of the option's life. In other words, the payoff of an ODB can only be obtained if at each instant of time before maturity the actual price of the underlying lies inside the range and this can only be achieved when the underlying lies all the time inside the corridor. Therefore the ODB may be considered as to be path-independent. For this reason, in order to price an ODB it suffices to consider $g$, given by eq. (4), instead of $\psi$. Consequently, the payoff function $\mathrm{g}$ is path-independent, and an ODB may be priced by means of Theorem 4.1.

It remains to specify the boundary conditions of the payoff of an ODB. The option vanishes when the underlying hits either the upper or the lower barrier, $H^{+}$or $H^{-}$. Thus, for all $t \leq T$ the following boundary conditions must hold for the pricing formula $f$ :

together with the final condition

$$
f\left(H^{-}, t\right)=0, \quad f\left(H^{+}, t\right)=0,
$$

$$
f(S, T)=R .
$$

In order to transform the Black-Scholes PDE into the heat equation, the solution of which is well known, we proceed as follows. First the PDE is made dimensionless by scaling $S$ and $f$ by the constant $H^{-}$. Then the backward PDE is turned into a forward PDE by reversing the time scale. Both steps are pursued by means of the following transformations where $x$ and $\tau$ are the new transformed variables: 


$$
\begin{gathered}
S=H^{-} e^{x}, \\
t=T-\frac{2 \tau}{\sigma^{2}}, \\
f(S, t)=H^{-} e^{\alpha \ln \left(\frac{S}{H^{-}}\right)+\beta(T-t) \frac{\sigma^{2}}{2}} u\left(\ln \left(\frac{S}{H^{-}}\right),(T-t) \frac{\sigma^{2}}{2}\right),
\end{gathered}
$$

or in shorthand notation:

$$
f=H^{-} e^{\alpha x+\beta \tau} u(x, \tau)
$$

where the constants $\alpha$ and $\beta$ are defined as

$$
\alpha:=-\frac{1}{2}(k-1) \quad \text { and } \quad \beta:=-\frac{1}{4}(k-1)^{2}-\frac{2}{\sigma^{2}} r_{d} \text {. }
$$

Inserting these substitutions into the Black-Scholes PDE indeed yields the heat equation, extensively investigated in the literature: (Note 5)

$$
\frac{\partial^{2} u}{\partial x^{2}}=\frac{\partial u}{\partial \tau}
$$

for $\tau>0$ and $0<x<\ln \left(\frac{H^{+}}{H^{-}}\right)$. Note that eq. (8) is a linear second order PDE. In fact, it belongs to the class of parabolic differential equations. (See, for example, Evans, 1998, p. 349 ff.)

The boundary conditions $f\left(H^{-}, t\right)=0$ and $f\left(H^{+}, t\right)=0$ have to be transformed as well. Using the transformed variables, given in eqs (5)-(7), the boundaries turn into

$$
u(0, \tau)=0, \quad u(L, \tau)=0
$$

for $\tau \geq 0$, where we define $L:=\ln \left(\frac{H^{+}}{H^{-}}\right)$as a shorthand notation. The final condition $f(S, T)=R$ turns under the same transformations into the following initial condition

$$
u(x, 0)=\frac{R e^{-\alpha x}}{H^{-}}
$$

for $0<x<L$.

We are now well prepared to derive the pricing formula of the ODB within the Black-Scholes model. — We skip, however, the derivation, which can be found in Ebenfeld, Mayr and Topper (2002), and state their result immediately. (Note 6)

Lemma 4.1 In the Black-Scholes model the arbitrage-free price of an ODB option $f$ is given by 


$$
\begin{gathered}
f(S, t)=\sum_{n=1}^{\infty} \frac{2 \pi n R}{L^{2}}\left[\frac{\left(\frac{S}{H^{-}}\right)^{\alpha}-(-1)^{n}\left(\frac{S}{H^{+}}\right)^{\alpha}}{\alpha^{2}+\left(\frac{n \pi}{L}\right)^{2}}\right] \sin \left(\frac{n \pi}{L} \ln \frac{S}{H^{-}}\right) \\
\quad \times \exp \left(-\frac{1}{2}\left[\left(\frac{n \pi}{L}\right)^{2}-\beta\right] \sigma^{2}(T-t)\right) .
\end{gathered}
$$

Thus far we have derived a pricing formula for ODB options. Since we have seen in Section 3 that an Onion option may be decomposed into three ODB options, $f_{\text {Onion }}=\sum_{i=1}^{3} f_{\text {odb }}\left(H_{i}^{+}, H_{i}^{-}\right)$, it remains to reverse this decomposition. To this end, it remains to substitute the ODB's pricing formula (9) on the right hand side and then we obtain the pricing formula for Onion options:

Theorem 4.2 In the Black-Scholes model the arbitrage-free price of an Onion option $f_{\text {Onion }}$ is given by

$$
\begin{aligned}
f_{\text {Onion }}(S, t)=\sum_{i=1}^{3} & \sum_{n=1}^{\infty}\left\{\frac{2 \pi n R}{L^{2}}\left[\frac{\left(\frac{S}{H_{i}^{-}}\right)^{\alpha}-(-1)^{n}\left(\frac{S}{H_{i}^{+}}\right)^{\alpha}}{\alpha^{2}+\left(\frac{n \pi}{L}\right)^{2}}\right]\right. \\
& \left.\times \sin \left(\frac{n \pi}{L} \ln \frac{S}{H_{i}^{-}}\right) \exp \left(-\frac{1}{2}\left[\left(\frac{n \pi}{L}\right)^{2}-\beta\right] \sigma^{2}(T-t)\right)\right\} .
\end{aligned}
$$

\section{An Alternative Approach to Price ODBs}

In this section an alternative approach for pricing ODBs and, therefore, Onion options is presented. Our starting point is the observation that the value of an ODB option may be viewed as the probability of the underlying price staying within the barriers during the option's life, discounted at $r_{d}$ and $r_{f}$. This encourages us to follow a probabilistic approach based on directly computing the risk-neutral valuation formula for currency options (A.1). A similar approach is also done by Geman and Yor Geman and Yor (1996) who price double barrier options via a probabilistic approach. Kunitomo and Ikeda (1992) price double barrier options with curved boundaries via a suitable probability measure. Our approach rest upon their work. - Eventually, we feel that this probabilistic approach is more intuitive than the transformation of a suitable version of the Black Scholes PDE into the heat equation.

Note that even though we are able to show that the pricing formula derived from the Black-Scholes PDE is unique, it is still possible to find another formula. However, a different approach must be chosen. The uniqueness theorem covers only solutions derived by solving the Black-Scholes PDE leaving the door open for alternative ways. Clearly, an alternative formula must yield the same value of an Onion option. Hence, a new formula may be found, but not a new price.

Recall that the risk-neutral valuation formula states that the option's arbitrage price equals its discounted expected payoff

$$
\pi_{t}(X)=e^{r_{d} t} \mathbb{E} \mathbb{P} \cdot\left[e^{-r_{d} T} X \mid \mathcal{F}_{t}\right],
$$

where $X$ is the option's payoff function. Yet, in order to use this formula, it is useful to have a more compact version of the payoff function than the one in eq. (1). This may be achieved by using the indicator function $\mathbf{1}_{\{\cdot\}}$ :

$$
\varphi_{i}\left(S_{T}\right)=R 1_{\left\{H_{i}^{-}<m(T), M(T)<H_{i}^{+}\right\}}, \quad i=1,2,3
$$

Note that although we wish to evaluate the Onion option, the payoff of which is given by

$$
\psi\left(S_{T}\right)=\varphi_{1}\left(S_{T}\right)+\varphi_{2}\left(S_{T}\right)+\varphi_{3}\left(S_{T}\right),
$$


it is sufficient to derive a valuation formula for the ODB, because the conditional expectation is linear and we, thus, obtain

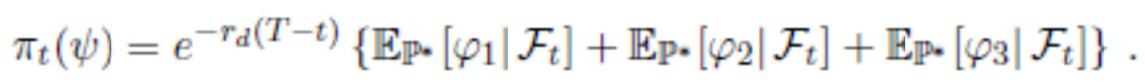

Substituting $\varphi_{i}$ in the risk-neutral valuation formula yields

$$
\left.\pi_{t}\left(\varphi_{i}\right)=R e^{-r_{d}(T-t)} \mathbb{E}_{\mathbb{P} \cdot\left[\mathbf{1}_{\left\{H_{i}^{-}<m(T)\right.}, M(T)<H_{i}^{+}\right\}} \mid \mathcal{F}_{t}\right] .
$$

In order to calculate this conditional expectation we define an auxiliary process $\hat{S}$. First, however, we recall from the literature that under the martingale measure $\mathbb{P}^{*}$ the process $S$ can also be written as

$$
S(u)=S(t) \exp \left(\sigma(W(u)-W(t))+\left(r-\frac{1}{2} \sigma^{2}\right)(u-t)\right),
$$

for all $u \geq t$, where $r$ is a shorthand notation for $\left(r_{d}-r_{f}\right)$. Note that since we are primarily concerned about currency options, both the domestic and the foreign interest rate together determine the drift of the process $S$. Now this alternative formulation for $S(u)$ is utilized in the definition of $\hat{S}$. The respective new auxiliary Wiener process $\hat{W}$ is defined for a fixed $t>0$ as

$$
\hat{W}(u):=W(t+u)-W(t), \quad \text { for } \quad u \geq 0 .
$$

The process $\hat{S}(u)$ is defined the following way

$$
\hat{S}(u):=\exp \left(\left(r-\frac{1}{2} \sigma^{2}\right) u+\sigma \hat{W}(u)\right) .
$$

It is easy to verify that with the help of $\hat{S}(t), S(u)$ can be written as

$$
S(u)=S(t) \hat{S}(u-t) \quad \text { for } \quad u \geq t \geq 0 .
$$

Observe that $\hat{S}(0)=1$.

Next we show in which way the maximum of the new process depends on $M(T)$, the maximum of the old process.

For a fixed point in time $t \leq T$, the maximum $M(T)$, can be rewritten with he help of $\hat{S}(t)$ 


$$
\begin{aligned}
M(T) & =\max _{u \leq T} S(u) \\
& =\max \left\{\max _{u \leq t} S(u), \max _{t<u \leq T} S(u)\right\} \\
& =\max \left\{M(t), \max _{t \leq u \leq T} S(t) \hat{S}(u-t)\right\} \\
& =\max \left\{M(t), S(t) \max _{t \leq u \leq T} \hat{S}(u-t)\right\} \\
& =\max \{M(t), S(t) \underbrace{\max _{0 \leq u \leq T} \hat{S}(u)}_{=: \hat{M}(T-t)}\} .
\end{aligned}
$$

A corresponding equation holds for the minimum, and we thus define the minimum of the process $\hat{S}$, denoted by $\hat{m}(T-t)$, analogously. Using this result the ODB's payoff function ${ }^{\varphi}$ may be rewritten as

$$
\begin{aligned}
\varphi & =R 1_{\left\{H^{-}<m(T), M(T)<H^{+}\right\}} \\
& =R 1_{\left\{H^{-}<m(t), M(t)<H^{+}\right\}} \mathbf{1}_{\left\{H^{-} / S(t)<\hat{m}(T-t), \hat{M}(T-t)<H^{+} / S(t)\right\}} .
\end{aligned}
$$

This new version of the payoff function is substituted in the risk-neutral valuation formula, yielding

$$
\begin{aligned}
\pi_{t}\left(\varphi(S(T))=R e^{-r_{d}(T-t)} \mathbb{E}_{\mathbb{P}}\right. & {\left[\mathbf{1}_{\left\{H^{-}<m(t), M(t)<H^{+}\right\}}\right.} \\
& \left.\times \mathbf{1}_{\left\{H^{-} / S(t)<\hat{m}(T-t), \hat{M}(T-t)<H^{+} / S(t)\right\}} \mid \mathcal{F}_{t}\right] \\
= & R e^{-r_{d}(T-t)} \mathbb{E}_{\mathbb{P} *}\left[1_{\left\{H^{-} / S(t)<\hat{m}(T-t), \hat{M}(T-t)<H^{+} / S(t)\right\}}\right] \\
= & R e^{-r_{d}(T-t)} \mathbb{P}^{*}\left[\frac{H^{-}}{S(t)}<\hat{m}(T-t), \hat{M}(T-t)<\frac{H^{+}}{S(t)}\right] .
\end{aligned}
$$

The first equality holds because $S(t), M(t)$ and $m(t)$ are $\mathcal{F}_{t^{-}}$measurable. Moreover, $\hat{M}(T-t)$ and $\hat{m}(T-t)$ are independent of the $\sigma$-algebra $\mathcal{F}_{t}$. (Note 7)

We define the price of an ODB option $g:\left[H^{-}, H^{+}\right] \times[0, T] \rightarrow \mathbb{R}$ as a function of the current spot price $S_{t}$ of the underlying and the time $t \in[0, T]$

$$
g\left(S_{t}, t\right)=R e^{-r_{d}(T-t)} \mathbb{P}^{*}\left[\frac{H^{-}}{S_{t}}<\hat{m}(T-t), \hat{M}(T-t)<\frac{H^{+}}{S_{t}}\right] .
$$

In order to compute $\mathrm{g}$ we need a density function for two absorbing barriers, one above and the other below the current spot price of the underlying. The following theorem provides exactly this density function. It is taken from Kunitomo and Ikeda (1992). 
Theorem 5.1 Suppose ${ }^{\{Y(t)\}}$ follows the geometric Brownian with $Y(0)=Y_{0}$ and $I \subseteq[A, B]$. Then the probability that $A<m(t) \leq M(t)<B$ for all $t \in[0, \tilde{t}]$ and $S(\tilde{t}) \in I$ is

$$
P(I)=\int_{I}\left(\sum_{n=-\infty}^{+\infty} k_{n}(y)\right) \frac{d y}{y},
$$

where

$$
\begin{aligned}
k_{n}(y)= & \left(\frac{B}{A}\right)^{n\left(\frac{2 r}{\sigma^{2}}-1\right)} c\left(\frac{\ln (y)-\ln \left(Y_{0}\left(\frac{B}{A}\right)^{2 n}\right)-\left(r-\frac{\sigma^{2}}{2}\right) \tilde{t}}{\sigma \sqrt{\tilde{t}}}\right) \\
& -\left(\frac{A^{n+1}}{Y_{0} B^{n}}\right)^{\frac{2 r}{\sigma^{2}}-1} c\left(\frac{\ln (y)-\ln \left(\left(\frac{A}{B}\right)^{2 n} \frac{A^{2}}{Y_{0}}\right)-\left(r-\frac{\sigma^{2}}{2}\right) \tilde{t}}{\sigma \sqrt{\tilde{t}}}\right)
\end{aligned}
$$

with $c(\cdot)$ being the density function of the normal distribution.

Proof: For the proof see the appendix of Kunitomo and Ikeda (1992). In order to get straight barriers we have to substitute $\delta_{1}=\delta_{2}=0$ in their proof.

Now the required density function is found and we thus can calculate the conditional expectation in the risk-neutral valuation formula of the Onion option. Note that the underlying process is now the auxiliary one $\hat{S}(u)$ and, therefore, we have $\hat{S}(0)=1$. Furthermore, the upper barrier is $\frac{H^{+}}{S_{t}}$ and the lower one is $\frac{H^{-}}{S_{t}}$ where $S_{t}$ is $\mathcal{F}_{t^{-}}$measurable. The time interval in which $\hat{S}(u)$ is defined is $[0, T-t]$. The interval $I$ is, of course, $\left(\frac{H^{-}}{S_{t}}, \frac{H^{+}}{S_{t}}\right)$.

Formally this yields

$$
g\left(S_{t}, t\right)=R e^{-r_{d}(T-t)} \int_{\frac{H^{-}}{S_{t}}}^{\frac{H^{+}}{S_{t}}}\left(\sum_{n=-\infty}^{+\infty} k_{n}(y)\right) \frac{d y}{y} .
$$

Hence, we obtain

$$
\begin{aligned}
g\left(S_{t}, t\right)= & R e^{-r_{d}(T-t)} \\
\times \sum_{m=-\infty}^{\infty} & \left\{\left(\frac{H^{+}}{H^{-}}\right)^{m\left(\frac{2}{\sigma^{2}}\left(r_{d}-r_{f}\right)-1\right)}\left(\mathcal{N}\left[d\left(H^{-}, m\right)\right]-\mathcal{N}\left[d\left(H^{+}, m\right)\right]\right)\right. \\
& -\left(\frac{H^{-}}{S_{t}}\right)^{\left(\frac{2}{\sigma^{2}}\left(r_{d}-r_{f}\right)-1\right)}\left(\frac{H^{-}}{H^{+}}\right)^{m\left(\frac{2}{\sigma^{2}}\left(r_{d}-r_{f}\right)-1\right)} \\
\times & \left.\left(\mathcal{N}\left[d\left(H^{-}, m\right)+z(m)\right]-\mathcal{N}\left[d\left(H^{+}, m\right)+z(m)\right]\right)\right\}
\end{aligned}
$$


where $\mathcal{N}[\cdot]$ stands again for the standard normal distribution and the following shorthand notations are used

$$
\begin{aligned}
d(X, m) & =\frac{\ln \left(\frac{S_{t}}{X}\right)+2 m \ln \left(\frac{H^{+}}{H^{-}}\right)+\left(\left(r_{d}-r_{f}\right)-\frac{\sigma^{2}}{2}\right)(T-t)}{\sigma \sqrt{(T-t)}}, \\
z(m) & =\frac{2 \ln \left(\frac{H^{-}}{S_{t}}\right)-4 m \ln \left(\frac{H^{+}}{H^{-}}\right)}{\sigma \sqrt{(T-t)}} .
\end{aligned}
$$

We have thus arrived at a pricing formula for ODB options. In order to obtain a pricing formula for the Onion option we just have to add three ODB pricing formulas with the respective barriers in the same way as we did in section 4 .

\section{Examples}

In this section we use the previously derived formulas (10) and (11) in order to provide two examples. These are used to compare the actual spot prices of an Onion option with its theoretical value in the Black-Scholes model. In order to calculate the latter, either of both formulas may be applied, as both must yield the same value.

As a first example consider the Onion option with the WKN 683989 (ISIN DE000683989/7) which is based on the USD/Euro exchange rate. This option expires on June 23rd, 2003 (Note 8) and it has these barriers

$$
\begin{array}{lll}
H_{1}^{-}=0.88, & H_{2}^{-}=0.87, & H_{3}^{-}=0.86, \\
H_{1}^{+}=1.06, & H_{2}^{+}=1.07, & H_{3}^{+}=1.08 .
\end{array}
$$

Table 1. Example with WKN: 683689 Comparison of the actual spot price and theoretical value of an Onion option with the USD/Euro exchange rate as underlying.

\begin{tabular}{llllllll}
\hline$t$ & $S_{t}$ & $\sigma$ & $r_{d}$ & $r_{f}$ & price & $f\left(S_{t}, t\right)$ & $\varepsilon$ \\
\hline $18 / 10 / 02$ & 0.9719 & $9.4113 \%$ & $2.50 \%$ & $1.5 \%$ & 18.610 & 18.7253 & $-0.34 \%$ \\
$25 / 10 / 02$ & 0.9778 & $9.0635 \%$ & $2.50 \%$ & $1.5 \%$ & 19.666 & 19.5221 & $+0.73 \%$ \\
$31 / 10 / 02$ & 0.9869 & $9.0473 \%$ & $2.50 \%$ & $1.5 \%$ & 18.350 & 18.9767 & $-3.41 \%$ \\
$04 / 11 / 02$ & 0.9951 & $8.9488 \%$ & $2.50 \%$ & $1.5 \%$ & 16.990 & 18.3347 & $-7.91 \%$ \\
$08 / 11 / 02$ & 1.0100 & $9.1379 \%$ & $2.50 \%$ & $1.5 \%$ & 15.020 & 15.4963 & $-3.17 \%$ \\
\hline $15 / 11 / 02$ & 1.0045 & $8.8538 \%$ & $2.50 \%$ & $1.5 \%$ & 16.600 & 17.4627 & $-5.20 \%$ \\
$20 / 11 / 02$ & 1.0021 & $8.8193 \%$ & $2.50 \%$ & $1.5 \%$ & 17.900 & 18.2201 & $-1.82 \%$ \\
$25 / 11 / 02$ & 0.9950 & $8.7629 \%$ & $2.50 \%$ & $1.5 \%$ & 20.230 & 19.8067 & $+2.09 \%$ \\
$02 / 12 / 02$ & 0.9931 & $8.4754 \%$ & $2.50 \%$ & $1.5 \%$ & 20.370 & 21.0924 & $-3.55 \%$ \\
$05 / 12 / 02$ & 1.0001 & $8.3665 \%$ & $2.50 \%$ & $1.5 \%$ & 19.640 & 20.3655 & $-3.69 \%$ \\
\hline $10 / 12 / 02$ & 1.1032 & $8.3937 \%$ & $2.50 \%$ & $1.5 \%$ & 17.530 & 17.7036 & $-1.01 \%$ \\
$16 / 12 / 02$ & 1.0215 & $8.3277 \%$ & $2.50 \%$ & $1.5 \%$ & 15.080 & 15.9169 & $-5.55 \%$ \\
$20 / 12 / 02$ & 1.0246 & $8.3547 \%$ & $2.50 \%$ & $1.5 \%$ & 14.290 & 15.1612 & $-2.83 \%$ \\
$02 / 01 / 03$ & 1.0474 & $8.4512 \%$ & $2.50 \%$ & $1.5 \%$ & 8.270 & 8.0362 & $+2.83 \%$ \\
$06 / 01 / 03$ & 1.0472 & $8.6746 \%$ & $2.50 \%$ & $1.5 \%$ & 8.730 & 8.0156 & $+8.18 \%$ \\
\hline $10 / 01 / 03$ & 1.0519 & $8.7311 \%$ & $2.50 \%$ & $1.5 \%$ & 6.230 & 6.4316 & $-3.24 \%$ \\
$15 / 01 / 03$ & 1.0518 & $8.6822 \%$ & $2.50 \%$ & $1.5 \%$ & 6.590 & 6.6088 & $-0.29 \%$ \\
$20 / 01 / 03$ (Note 9$)$ & 1.0658 & $8.6585 \%$ & $2.50 \%$ & $1.5 \%$ & 2.670 & 2.2781 & $+14.68 \%$ \\
$22 / 01 / 03$ & 1.0690 & $8.5790 \%$ & $2.50 \%$ & $1.5 \%$ & 1.580 & 1.5071 & $+4.61 \%$ \\
\hline
\end{tabular}


The obtained results are provided in Table 1 . The last column denotes the relative difference between the real and theoretical value which is defined as $\varepsilon=($ price $-f)$ /price. This is a suitable measure for the difference because it makes them comparable for different spot prices. In this example $\varepsilon$ is almost always between $-5 \%$ and $5 \%$ with only few exceptions. Hence, most of the time the actual price is very close to the theoretical value.

As a second example we consider an Onion option with the Yen/Euro exchange rate as an underlying: WKN 683986 (ISIN DE000683896/3). This option expires on December 22nd, 2003 and its barriers are (Note 10)

$$
\begin{array}{lll}
H_{1}^{-}=110, & H_{2}^{-}=109, & H_{3}^{-}=108, \\
H_{1}^{+}=130, & H_{2}^{+}=131, & H_{3}^{+}=132 .
\end{array}
$$

Table 2. Example with WKN: 683686 Comparison of the actual spot price and theoretical value of an Onion option with the Yen/Euro exchange rate as underlying

\begin{tabular}{llllllll}
\hline$t$ & $S_{t}$ & $\sigma$ & $r_{d}$ & $r_{f}$ & price & $f\left(S_{t}, t\right)$ & $\varepsilon$ \\
\hline $09 / 10 / 02$ & 121.7019 & $7.8039 \%$ & $2.50 \%$ & $0.1 \%$ & 8.65 & 10.9946 & $-27.11 \%$ \\
$23 / 10 / 02$ & 121.5562 & $8.7051 \%$ & $2.50 \%$ & $0.1 \%$ & 8.87 & 11.8035 & $-33.43 \%$ \\
$06 / 11 / 02$ & 121.9740 & $7.3572 \%$ & $2.50 \%$ & $0.1 \%$ & 10.38 & 12.7964 & $-23.28 \%$ \\
$20 / 11 / 02$ & 122.6710 & $7.4478 \%$ & $2.50 \%$ & $0.1 \%$ & 10.71 & 12.2276 & $-14.17 \%$ \\
$27 / 11 / 02$ & 121.6544 & $7.8869 \%$ & $2.50 \%$ & $0.1 \%$ & 11.28 & 12.2992 & $-9.04 \%$ \\
\hline $11 / 12 / 02$ & 124.6015 & $7.2392 \%$ & $2.50 \%$ & $0.1 \%$ & 9.45 & 10.6507 & $-12.71 \%$ \\
$27 / 12 / 02$ & 124.5606 & $7.3176 \%$ & $2.50 \%$ & $0.1 \%$ & 9.32 & 10.9937 & $-17.96 \%$ \\
$08 / 01 / 03$ & 125.0861 & $7.0877 \%$ & $2.50 \%$ & $0.1 \%$ & 9.97 & 10.8285 & $-8.61 \%$ \\
$22 / 01 / 03$ & 126.5813 & $7.0960 \%$ & $2.50 \%$ & $0.1 \%$ & 8.43 & 8.3705 & $+0.71 \%$ \\
$29 / 01 / 03$ & 128.5304 & $6.9938 \%$ & $2.50 \%$ & $0.1 \%$ & 6.49 & 4.7324 & $+27.08 \%$ \\
\hline $12 / 02 / 03$ (Note 11$)$ & 129.8147 & $6.9912 \%$ & $2.50 \%$ & $0.1 \%$ & 3.07 & 2.8183 & $+8.20 \%$ \\
$19 / 02 / 03$ & 127.4619 & $7.1982 \%$ & $2.50 \%$ & $0.1 \%$ & 5.74 & 6.7933 & $-18.35 \%$ \\
$05 / 03 / 03$ & 128.8728 & $7.3143 \%$ & $2.50 \%$ & $0.1 \%$ & 4.49 & 4.6102 & $-2.68 \%$ \\
$12 / 03 / 03$ & 129.4159 & $7.2765 \%$ & $2.50 \%$ & $0.1 \%$ & 4.32 & 3.7576 & $+13.02 \%$ \\
$26 / 03 / 03$ & 128.2362 & $7.4387 \%$ & $2.50 \%$ & $0.1 \%$ & 5.56 & 6.1392 & $-10.42 \%$ \\
\hline $09 / 04 / 03$ & 128.6894 & $7.4674 \%$ & $2.50 \%$ & $0.1 \%$ & 4.81 & 5.5360 & $-15.09 \%$ \\
$22 / 04 / 03$ & 131.6842 & $7.4721 \%$ & $2.50 \%$ & $0.1 \%$ & 0.97 & 0.2277 & $+76.53 \%$ \\
\hline
\end{tabular}

The results obtained for this option are displayed in Table 2. The relative deviation of the actual prices from the theoretical prices are more substantial than in the first example. Arguably the assumptions underlying the Black-Scholes model are not justified in the present context: Transaction cost may make potential arbitrage opportunities vanishing; investors may be barred from replicating and trading exotic options. And also, while arbitrage opportunities did exist for some time, no trader may have realized this.

Now consider an accompanying Onion option, the option with the WKN 683 983. It has the same underlying and the same barriers as the option in the last example. The only difference between the two is the expiration date. While the option of our previous example expires on December 22nd, 2003, this option expires already three months earlier on September 22nd, 2003. Some of their respective actual spot prices were:

\begin{tabular}{llllll}
\hline WKN & expiration & $3 / 10 / 02$ & $23 / 10 / 02$ & $13 / 11 / 02$ & $27 / 11 / 02$ \\
\hline 683986 & $22 / 12 / 03$ & 9.42 & 8.87 & 10.34 & 11.28 \\
\hline 686983 & $22 / 09 / 03$ & 7.19 & 7.02 & 8.33 & 9.38 \\
\hline
\end{tabular}

In fact, these prices provided an arbitrage possibility: Sell the longer-running option short, and buy the same number of options of the cheaper, shorter-running option. The difference between the two spot prices is already a save profit which can be invested in a risk free bond. The following scenarios might now happen: Either the options reach the third barrier, then both options expire worthless. However, that happens only simultaneously as they have exactly the 
same barriers. In this case, there is still the margin from the beginning - a riskless profit. In the other scenario the long position yields a positive payoff at maturity, the exact amount of which of course depends on the barriers which were hit. Yet, irrespective of which barriers are hit, the options in the short position have reached the same barriers and can, therefore, not yield a higher payoff. Therefore, use the payoff of the short-running option to either settle the short position at maturity, or if it expires worthless before maturity, an additional profit is collected. - Remarkably, this arbitrage opportunity lasted for almost two months until at least the relative price difference was reduced in the market.

This type of mispricing is hard to explain within the Black-Scholes model: There is no reason for any investor to buy the longer-running option, and the emitting bank runs the risk of potential losses if customers become aware of this arbitrage opportunity. In fact, if someone had detected this opportunity, prices should have adjusted quickly. Since this obviously did not occur, we may conclude that the Black-Scholes model is only of limited scope within this framework. In particular, within the Black-Scholes model, it is assumed that the market is frictionless, implying that, among other things, a single deal has no direct effect on the option's price. However, the thinner the trading volume is, the more unrealistic is this assumption - and notably exotic derivatives frequently have a thin trading volume. Also, the Black-Scholes model may simply not be applicable, and correspondingly the issuer may have decided not to price the option according to the arbitrage-free price within the Black-Scholes model.

\section{Conclusions}

In this paper we have considered a particular type of an exotic option: Onion options. This type of an option may be viewed as a modified double barrier option: it has several corridors and each time the price of the underlying touches either the upper or the lower barrier of a corridor, the payoff of the option is reduced by some given amount. If up to the time of maturity of the option, the barriers of all corridors are touched, the option ceases to exist and the payoff is nil, otherwise the payoff is determined by those corridors the barriers of which are not hit.

Although the payoff of an Onion option is determined by a simple rule, their valuation is by no means simple. In order to obtain an arbitrage-free formula within the Black-Scholes model, two features turn out to be of significant importance. Firstly, an Onion option may be decomposed into several one-touch double barrier binary options (ODB); and secondly, both the Onion option and the ODB may be regarded as to be path independent as their payoff does not directly depend on the path of the underlying provided that the option is alive at maturity. Given these two features one can derive pricing formulas for the Onion option. Ebenfeld, Mayr, and Topper (2002) provide such a formula based on the well known Black-Scholes PDE.

We recapitulate this result and then provide an alternative pricing rule based upon the risk-neutral valuation formula for currency options. Although both formulas are derived in different ways and look differently — in fact both formulas require the calculation of an infinite sum - , the prices they yield for a given option necessarily coincide. So both the formula of Ebenfeld, Mayr, and Topper as well as ours allow for calculating the arbitrage-free price of an Onion option, and then to compare this price with the actual spot market price.

A few questions are left open for further research. One is to scrutinize the numerical behaviour of both valuation formulae, i.e., their speed of convergence, numerical stability etc. Moreover, it is also an open question how an Onion option can be hedged without using Onion options or other double barrier derivatives. Finally, one may modify the payoff structure of the Onion option somewhat so as to generate new, closely related products. For example, one may consider an Onion option with knock-in effects instead of with knock-out effects.

\section{References}

Björk, Tomas. (2009). Arbitrage Theory in Continuous Time (3rd ed.). Oxford University Press.

Black, Fischer, \& Scholes, Myron. (1973). The Pricing of Options and Corporate Liabilities. Journal of Political Economics, 81(3), 637-54. http://dx.doi.org/10.1086/260062

Bleecker, David, \& Csordas, George. (1996). Basic Partial Differential Equations. International Press.

Buchen, Peter, \& Konstandatos, Otto. (2009). A New Approach to Pricing Double-Barrier Options with Arbitrary Payoffs and Exponential Boundaries. Applied Mathematical Finance, 16(5-6), 497-515. http://dx.doi.org/10.1080/13504860903075480

Chen, Chang-Chih, Wang, Chou-Wen, \& Shyu, David. (2010). Pricing Double Barrier Options Using Reflection Principle. International Research Journal of Finance and Economics, 37, 125-137.

Churchill, Ruel v., \& Brown, James Ward. (1978). Fourier Series and Boundary Value Problems (3rd ed.). McGraw-Hill. 
Ebenfeld, Stefan, Mayr, Matthias R., \& Topper, Jürgen. (2002). An Analysis of Onion Options and Double-no-Touch Digitals. Wilmott Magazine, 2, 68-77.

Evans, Lawrence C. (1998). Partial Differential Equations. American Mathematical Society.

Geman, H'elyette, \& Yor, Marc. (1996). Pricing and Hedging Double-Barrier Options: A Probabilistic Approach. Mathematical Finance, 6(4), 365-378. http://dx.doi.org/10.1111/j.1467-9965.1996.tb00122.x

Guillaume, Tristan. (2003). Window Double Barrier Options. Review of Derivatives Research, 6(1), 47-75. http://dx.doi.org/10.1023/A:1022874005113

Guillaume, Tristan. (2010). Step Double Barrier Options. Journal of Derivatives, 18(1), 59-79. http://dx.doi.org/10.3905/jod.2010.18.1.059

Hui, Cho H. (1996). One-Touch Double Barrier Option Values. Applied Financial Economics, 6, 343-346. http://dx.doi.org/10.1080/096031096334141

Hull, John C. (2000). Options, Futures and Other Derivatives (4th ed.). Prentice Hall International Editions.

Kunitomo, Naoto, \& Ikeda, Masayuki. (1992). Pricing Options with Curved Boundaries. Mathematical Finance, 2(4), 275-298. http://dx.doi.org/10.1111/j.1467-9965.1992.tb00033.x

Labart, Celine, \& Lelong, Jerome. (2009). Pricing Double Barrier Parisian Options Using Laplace Transforms. International Journal of Theoretical and Applied Finance, 12(1), 19-44. http://dx.doi.org/10.1142/S0219024909005154

Luo, Lawrence S. J. (2001). Various Types of Double-Barrier Options. Journal of Computational Finance, 4(3), 125-138.

Musiela, Marek, \& Rutkowski, Marek. (2005). Martingale Methods in Financial Modelling (2nd ed.). Springer.

Obloj, Jan, \& Ulmer, Frederik. (2012). Performance of Robust Hedges for Digital Double Barrier Options. International Journal of Theoretical and Applied Finance, 15(1), 1-34. http://dx.doi.org/10.1142/S0219024911006516

Zhang, Peter. (1998). Exotic Options: A Guide to Second Generation Options (2nd ed.). World Scientific.

\section{Notes}

Note 1. In 1998 Warburg Dillon Read was the first bank to offer an exotic option with these characteristics. They called it a Multi-Double-Lock-Out-Warrant and its underlying was a foreign exchange rate. Later they issued another option named Quattro-option - an Onion option with an additional corridor and once again a foreign exchange rate as underlying. Sometimes the Onion option is also called Trinity-Double-Lock-Out Warrant.

Note 2. For more information about barrier options see, for example, Zhang (1998) pp. 203-259.

Note 3. A binary option with a fixed monetary payoff is called cash-or-nothing option.

Note 4. The same idea is proposed by Ebenfeld, Mayr and Topper (2002).

Note 5. See for example Bleecker and Csordas (1996), Churchill and Brown (1978) or Evans (1998).

Note 6 . It is straightforward but tedious to check that $f$ satisfies the boundary and final conditions and that it converges.

Note 7. This can be seen the following way: $\hat{M}(T-t)=\max _{0<u<T-t} \hat{S}(u)$ and for every $0 \gg t \gg T$ holds $\hat{S}(T-t)=\exp \left(\left(r-\frac{\sigma^{2}}{2}\right)(T-t)+\sigma \hat{W}(T-t)\right)$ where $\hat{W}(T-t)=W(T)-W(t)$ and $W(T)-W(t)$ is independent of $\mathcal{F}_{t}$ by the definition of a Wiener process. Therefore, $\hat{M}(T-t)$ is as well as $\hat{m}(T-t)$ independent of $\mathcal{F}_{t}$.

Note 8. Actually, the first barrier of this option got knocked out on January 17th, 2003, and the option got ultimately knocked out on January 24th, 2003. 
Note 9. This first barrier of this option got knocked out on January 17th, 2003.

Note 10. Actually, the first barrier of this option got knocked out on February 5th, 2003, and the option got ultimately knocked out on April 23rd, 2003.

Note 11. This first barrier of this option got knocked out on February 5th, 2003.

\section{Appendix A. Currency Options}

As mentioned above, the underlying of an Onion option is frequently a foreign exchange. To this end we must complement our model by taking into account for the foreign currency, and thus for the foreign interest rate. In this section we modify the Black-Scholes model accordingly. In the Black-Scholes model stock price processes are usually assumed to follow a geometric Brownian motion - and this also assumed to be true for foreign exchange rates. (Confer for example Hull, 2000, p. 283 and Björk, 2009, ch. 17.)

We shall see that the effect of introducing a foreign interest rate, denoted by $r_{f}$, is similar to that of allowing for a stock to pay dividends. To this end, we extend the Black-Scholes model for dividend paying underlyings - or for currency options. This procedure leads us to a modification of the original Black-Scholes PDE of Theorem 4.1. The following lines are based on Musiela and Rutkowski (2005), ch. 3.2 and Björk (2009), ch. 16.2.

Subsequently we assume that the stock pays a continuous dividend at a fixed rate $\eta$ proportional to the value of the underlying. In order to account for this, we first have to generalize the concept of a self-financing strategy. Let $\phi=\left(\phi^{1}, \phi^{2}\right)$ be a trading strategy. Now $\phi$ is called self-financing if its wealth process satisfies the following condition

$$
d V_{t}(\phi)=\phi_{t}^{1} d S_{t}+\eta \phi_{t}^{1} S_{t} d t+\phi_{t}^{2} d B_{t}
$$

Next, we introduce an auxiliary process to describe the dividend paying stock. Defining $\mu_{\eta}=\mu+\eta$, the auxiliary process $\widetilde{S}_{t}$ is defined the following way

$$
\widetilde{S}_{t}=e^{\eta t} S_{t},
$$

where $S_{t}$ is the original stock price process (i. e. without a dividend). It is easy to verify that the dynamic of the auxiliary process is given by the stochastic differential equation

$$
d \widetilde{S}_{t}=\mu_{\eta} \widetilde{S}_{t} d t+\sigma \widetilde{S}_{t} d W_{t} .
$$

To obtain the modified Black-Scholes PDE we first need a modified risk neutral valuation formula for currency options.With $r_{d}$ denoting the domestic interest rate the following formula holds

$$
\pi_{t}(X)=e^{r_{d} t} \mathbb{E}_{\mathrm{p} \cdot}\left[e^{-r_{d} T} X \mid \mathcal{F}_{t}\right], \quad \forall t \in[0, T] .
$$

The difference to the original version of this formula is that the unique martingale measure $\mathbb{P}^{*}$ differs from the original one, and thus the stock follows a different dynamic.

The Black-Scholes PDE for dividend paying underlyings reads as

$$
\frac{\partial v}{\partial t}+\frac{1}{2} \sigma^{2} s^{2} \frac{\partial^{2} v}{\partial s^{2}}+(r-\eta) s \frac{\partial v}{\partial s}-r v=0,
$$


for all $(s, t) \in(0, \infty) \times(0, T)$, subject to the terminal condition

$$
v(s, T)=g(s) .
$$

The proof is basically the same as for Theorem 4.1 only replacing the underlying by $\widetilde{S}_{t}$ and its dynamic.

Thus, the Black-Scholes PDE for currency options is obtained

$$
\frac{\partial v}{\partial t}+\frac{1}{2} \sigma^{2} s^{2} \frac{\partial^{2} v}{\partial s^{2}}+\left(r_{d}-r_{f}\right) s \frac{\partial v}{\partial s}-r_{d} v=0,
$$

subject to the same terminal condition

$$
v(s, T)=g(s)
$$

where $g(s)$ is the option's payoff function. 